\title{
Original
}

\section{Decreased fasting blood glucose is associated with impaired hepatic glucose production in thyroid-stimulating hormone receptor knockout mice}

\author{
Tingting Wang ${ }^{1), 3)}$, Jin $\mathrm{Xu}^{1)}$, Tao $\mathrm{Bo}^{2)}$, Xiaoming Zhou ${ }^{2)}$, Xiuyun Jiang ${ }^{1), 3)}$, Ling $\mathrm{Gao}^{2), 3)}$ and Jiajun Zhao ${ }^{1), 3)}$ \\ 1) Department of Endocrinology, Provincial Hospital Affiliated to Shandong University, Jinan, China \\ 2) Central Laboratory, Provincial Hospital Affiliated to Shandong University, Jinan, China \\ 3) Institute of Endocrinology, Shandong Academy of Clinical Medicine, Jinan, China
}

\begin{abstract}
Our previous study reported that thyroid-stimulating hormone (TSH) promotes cholesterol synthesis via the cyclic adenosine monophosphate/protein kinase A/cAMP regulatory element-binding protein (cAMP/PKA/CREB) pathway after binding to TSH receptors (TSHR) in the liver. The hepatic cAMP/PKA/CREB pathway also plays an important role in maintaining fasting glucose homeostasis. These findings implied a possible role for TSH in hepatic glucose metabolism. In this study, we used TSH receptor knockout mice (Tshr-ko mice) to clarify the effect of $T s h r$ deletion on hepatic glucose metabolism, and investigated whether the effects of TSH directly regulate hepatic gluconeogenesis in HepG2 cells. Tshr-ko mice exhibited decreased fasting blood glucose levels, increased insulin sensitivity but normal level of fasting plasma insulin. Tshr deletion impaired hepatic glucose production by down-regulating the expression of glucose-6-phosphatase (G6P) and phosphoenolpyruvate pyruvate carboxylase (PEPCK) mRNA, two rate-limiting enzymes in hepatic gluconeogenesis, and enhancing the abundance of hepatic glucokinase (GK), the first enzyme regulating glycogen synthesis. Moreover, $T s h r$ deletion inhibited the protein expression of hepatic phospho-CREB and increased the protein expression of hepatic phospho-AMP-activated protein kinase (p-AMPK), two up-stream regulators of PEPCK and G6P mRNA. In HepG2 cells, TSH increased the expression of G6P and PEPCK at mRNA level. These results indicated the simulative effects of TSH on hepatic glucose production in vivo and in vitro, suggesting a novel role for TSH in hepatic glucose metabolism.
\end{abstract}

Key words: Thyroid-stimulating hormone receptors (TSHR), Gluconeogenesis, cAMP regulatory element binding protein (CREB), AMP-activated protein kinase (AMPK)

SUBCLINICAL HYPOTHYROIDISM (SCH) is characterized by an increased level of plasma TSH and normal levels of free thyroxin (FT4) and free triiodothyronine (FT3). With increasing evidence from clinical studies, the association between $\mathrm{SCH}$ and other diseases, such as hypercholesterolemia [1] and coronary heart disease [2], is receiving increasing attention. Some studies have also found associations between $\mathrm{SCH}$ and insulin resistance [3-5] or fasting hyperinsulinemia [6]. Amati [7] reported that $\mathrm{SCH}$ patients had significantly lower improvements in insulin sensitivity relative to euthyroid group under similar regular exercise and body weight loss. And some studies $[8,9]$ observed an improvement of insulin sensitivity when

Submitted Dec. 28, 2012; Accepted Apr. 2, 2013 as EJ12-0462 Released online in J-STAGE as advance publication May 10, 2013 Correspondence to: Jiajun Zhao, M.D., Ph.D., and Ling Gao, M.D., Provincial Hospital affiliated to Shandong University, 324 Jing 5 Road, Jinan, Shandong Province, 250021 China.

E-mail:jjzhao@medmail.com.cn(JZ)/gaoling1@medmail.com.cn(LG) the level of serum TSH was reduced within the normal range in $\mathrm{SCH}$ patients. Moreover, in the population of nondiabetic elderly men, it was reported that the TSHR-Asp727Glu polymorphism was associated with insulin resistance, suggesting that TSHR plays a role in glucose metabolism [10]. Taken together, these clinical studies strongly suggest a possible role for TSH in glucose metabolism.

TSH is synthesized and secreted by the pituitary gland and directly regulates thyroid function via binding to TSH receptor located in the membrane of thyrocytes. Recently, multiple studies have found that extrathyroidic tissues express TSHR [11]. Our previous studies [12, 13] demonstrated that functional TSHR was expressed

Abbreviations: TSH, thyroid-stimulating hormone; TSHR, TSH receptors; cAMP, cyclic adenosine monophosphate; PKA, protein kinase A; CREB, cAMP regulatory element binding protein; G6P, glucose 6-phosphatase; PEPCK, phosphoenolpyruvate pyruvate carboxylase; p-AMPK, phospho-AMP-activated protein kinase; GSK, glycogen synthase kinase; IGF-1, insulin-like growth factors 1; GK, glucokinase 
in hepatocytes, and the hepatic cAMP/PKA/CREB pathway was involved in the TSH-induced up-regulation of cholesterol synthesis. The hepatic cAMP/PKA/CREB pathway is also vital for regulating glucose metabolism in response to fasting. In the fasted state, hepatic glucose production is promoted (including gluconeogenesis and glycogenolysis) to maintain fasting glucose homeostasis via the cAMP/PKA/CREB pathway [14]. Therefore, abnormal regulation of the cAMP/PKA/CREB pathway could lead to variations in hepatic glucose production, which is vital for fasting blood glucose.

AMPK is considered to be a cellular monitor of energy charge that can regulate glucose metabolism in liver [15]. Once hepatic AMPK is activated, it can phosphorylate downstream substrates, such as glycogen synthase kinase (GSK) $3 \beta$ [16], to regulate hepatic glucose metabolism, including decreasing hepatic gluconeogenesis and glycogenolysis and enhancing glycogen synthesis. The evidence from Andrade's study showed that TSH could suppress AMPK activation in the thyroid gland [17]. Based on the above findings, we hypothesized that TSH may be involved in the regulation of hepatic glucose metabolism.

In this study, we used $T s h r$-ko mice as our experimental model to test whether Tshr deletion affect hepatic glucose metabolism in vivo and we also examined the effects of TSH on gluconeogenesis in vitro. Our findings indicated that $T s h r$ deletion could decrease fasting glucose and inhibit hepatic glucose production, and the possible mechanisms were related to p-CREB inhibition and AMPK activation.

\section{Materials and Methods}

\section{Animals and diets}

Wild-type $\left(T s h r^{+/+}\right)$mice and $T s h r$-ko $\left(T s h r^{-/}\right)$mice were obtained by breeding heterozygote $\left(T s h r^{+/}\right)$mice, which were purchased from Jackson Laboratory (USA). All mice were C57BL/6J-129S1/Sv hybrids. They had access to food and water ad libitum and were exposed to a 12-h light-dark cycle in a temperature-controlled room $\left(23^{\circ} \mathrm{C}\right)$. $T s h r$-ko mice were fed a diet containing $100 \mathrm{ppm}$ thyroid powder (Sigma) from 21 days-old [18]. When fed the supplemented diet for 3 weeks, 6-weekold wild-type mice and $T s h r$-ko mice were collected blood to measure the level of serum total T4 (TT4). On the experimental day, 8-week-old wild-type mice and $T s h r$-ko littermate mice were weighed and anesthetized with pentobarbital sodium after 8-hours fast. Blood samples were collected. The liver, heart and kidney were weighed, and the liver was divided into three parts. One part was frozen in liquid nitrogen until analysis, and the other two parts were separately fixed in $4 \%$ paraformaldehyde for hematoxylin and eosin (H\&E) staining or Carnoy's fluid for Periodic Acid Schiff (PAS) staining. All animal experiments were performed according to the Shandong University Institutional Animal Care and Use Committee (Jinan, China).

\section{Blood parameters}

The serum TT4, insulin, glucagon and insulin-like growth factors 1 (IGF-1) concentrations were measured using radioimmunoassay (Jiuding, Tianjing Biomedical Engineering limited company and HTA CO., LTD., China). The serum TSH level was assayed using an ELISA kit (Uscn Life Science Inc.). The blood glucose level was detected with an OneTouch Ultra glucometer (Johnson \& Johnson, USA). Insulin sensitivity was assessed with the insulin sensitivity index (ISI) as follows: $\mathrm{ISI}=1 /(\mathrm{FBG}(\mathrm{mmol} / \mathrm{L}) \times \mathrm{FINS}(\mathrm{mIU} / \mathrm{L}))[19]($ data were calculated after natural logarithmic transformation because this index was not normally distributed).

\section{Metabolic studies}

To evaluate the glucose metabolism in $T s h r$-ko mice, oral glucose tolerance tests (OGTT), insulin tolerance tests (ITT) and pyruvate tolerance tests (PTT) were performed as described previously [20, 21]. In brief, for OGTT, the mice were administered glucose $(2 \mathrm{~g} / \mathrm{kg}$ body weight) after an $18 \mathrm{~h}$ fast, and the levels of blood glucose were measured at $0,30,60$ and 120 minutes using a glucometer. For ITT, mice fasted for $5 \mathrm{~h}$ were injected intraperitoneally with insulin $(1.0 \mathrm{U} / \mathrm{kg}$ body weight, human insulin, Lily), and blood glucose levels were detected at $0,15,30,60$ and 90 minutes after insulin injection. For PTT, mice fasted for $18 \mathrm{~h}$ were injected intraperitoneally with pyruvate $(2 \mathrm{~g} / \mathrm{kg}$ body weight) dissolved in saline, and blood glucose were measured at $0,30,60$ and 90 minutes after pyruvate injection. The area under the curve (AUC) was calculated.

\section{Cell Culture}

The HepG2 human hepatoma cell line was purchased from Chinese Academy of Science (Shanghai). HepG2 cells were cultured in Eagle's minimum essential medium (EMEM) (GIBCO) containing 10\% fetal bovine serum and $100 \mathrm{U} / \mathrm{mL}$ penicillin-streptomycin in a humidified incubator of $95 \%$ air and 5\% CO2 at 
$37^{\circ} \mathrm{C}$. When the cells reached $80-90 \%$ confluence, the cells were exposed to $0.2 \mu \mathrm{m} \mathrm{TSH}$ for $20 \mathrm{~h}$ under serum-free medium.

$R N A$ extraction and quantitative real-time PCR analysis

Total RNA was extracted from HepG2 cells and mouse liver tissue using Trizol (TAKARA), and single-strand cDNA was synthesized from $5 \mu \mathrm{g}$ of total RNA with random hexamer primers (TAKARA) in a $10-\mu \mathrm{L}$ final volume. Real-time PCR was carried out with SYBR Premix Ex Taq II (TAKARA) in a final volume of $20 \mu \mathrm{L}$ containing $250 \mathrm{ng}$ of reverse-transcribed total RNA, $400 \mathrm{nM}$ of primers, and $10 \mu \mathrm{L}$ of $2 \times$ SYBR Premix Ex Taq II. PCR was performed for 40 cycles at $95^{\circ} \mathrm{C}$ for $15 \mathrm{sec}, 60^{\circ} \mathrm{C}$ for $30 \mathrm{sec}$, and $72{ }^{\circ} \mathrm{C}$ for $30 \mathrm{sec}$ in a LightCycler instrument (Roche Diagnostics). We determined the relative quantification of gene expression by the $2^{-\triangle \Delta C T}$ method [22]. The sense and antisense PCR primers used are listed in Table 1.

\section{Western blot analysis}

Liver tissue samples were homogenized in total protein extract solution and separated by centrifugation after sufficient lysis. The supernatant was collected, and the protein concentration was determined using the BCA method. Proteins $(100 \mu \mathrm{g})$ were separated using SDS-PAGE with $12 \%$ polyacrylamide gels and transferred to PVDF membranes (Millipore, Billerica, MA, USA). The membranes were blocked $(0.5 \%$ non-fat milk powder in TBST) for $1 \mathrm{~h}$ before incubation with primary antibodies at $4{ }^{\circ} \mathrm{C}$ overnight. The primary antibodies: phospho-CREB (Ser-133) (1:1000), CREB (1:1000), phospho-GSK3 $\beta$ (Ser-9) (1:1000), GSK3 $\beta$ (1:1000), phospho-AMPK (Thr-172) (1:1000),
AMPK (1:1000) and $\beta$-actin (1:10000) were from Cell Signaling Technology (Boston, USA). After incubation with either anti-rabbit or anti-mouse secondary antibody, the immune complexes were detected. The relative target protein levels were normalized to $\beta$-actin in the same membrane.

\section{Histology and PAS staining}

Liver paraffin-embedded tissues were sectioned at 5 $\mu \mathrm{m}$ and stained with H\&E. For hepatic glycogen staining, samples were stained with Periodic Acid Schiff according to the manufacturer's instructions (Yili Company, Beijing, China).

\section{Hepatic glycogen assay}

The hepatic glycogen content was assayed as described previously [23]. Briefly, $100 \mathrm{mg}$ liver was homogenized in $1.5 \mathrm{~mL}$ of $30 \% \mathrm{KOH}$ and dissolved at $100^{\circ} \mathrm{C}$ for $15 \mathrm{~min}$. The glycogen content was determined by adding $2.5 \mathrm{~mL}$ anthrone reagent $(2 \mathrm{mg}$ anthrone $/ \mathrm{mL}$ sulfuric acid) and measuring the absorbance at $620 \mathrm{~nm}$. Glucose solution was used as a standard.

\section{Statistics}

The data are presented as the mean \pm SD. Statistical significance was tested with Student's $t$ test and analysis of variance of repeated data with SPSS 17.0. Differences were considered statistically significant at $p<0.05$.

\section{Results}

\section{Tshr-ko mice exhibit decreased fasting blood glucose but normal plasma insulin level}

When fed the non-supplemented diet, Tshr-ko mice

Table 1 Primer sequences used for real-time PCR analysis

\begin{tabular}{|c|c|c|c|c|}
\hline & Gene name & Accession No. & Primer sequence & Amplicon length (bp) \\
\hline \multirow{6}{*}{ HepG2 cells } & PEPCK & NM_002591 & 5'-GAAAAAACCTGGGGCACAT-3'(forward) & 155 \\
\hline & & & 5'-TTGCTTCAAGGCAAGGATCTCT-3'(reverse) & \\
\hline & G6P & NM_001270397 & 5'-GACCTCAGGAATGCCTTCTACG-3'(forward) & 166 \\
\hline & & & 5'-AGTCAGTATCCAAAACCCACCAG-3' (reverse) & \\
\hline & $\beta$-actin & NM_001101.3 & 5'- ACAGAGCCTCGCCTTTGCCG -3'(forward) & 104 \\
\hline & & & 5'- ACATGCCGGAGCCGTTGTCG -3' (reverse) & \\
\hline \multirow{8}{*}{ Mice } & PEPCK & NM_011044 & 5'-GTGCTGGAGTGGATGTTCGG-3'(forward) & 258 \\
\hline & & & 5'-CTGGCTGATTCTCTGTTTCAGG-3'(reverse) & \\
\hline & G6P & ВC013448 & 5'-ACTGTGGGCATCAATCTCCTC-3'(forward) & 344 \\
\hline & & & 5'-CGGGACAGACAGACGTTCAGC-3' (reverse) & \\
\hline & GK & NM_010292 & 5'-GAAGACCTGAAGAAGGTGATGAGC-3'(forward) & 399 \\
\hline & & & 5'-GTCTATGTCTTCGTGCCTTACAGG-3' (reverse) & \\
\hline & $\beta$-actin & NM_007393 & 5'-ACCCCAGCCATGTACGTAGC-3'(forward) & 101 \\
\hline & & & 5'-GTGTGGGTGACCCCGTCTC-3' (reverse) & \\
\hline
\end{tabular}


exhibited decreased serum T4/T3 levels and elevated TSH level [18]. Therefore, in our study, all Tshr-ko mice were fed a diet containing $100 \mathrm{ppm}$ thyroid powder to eliminate the effect of abnormal thyroid hormone levels on glucose metabolism. As shown in Table 2, 6-week-old and 8-week-old Tshr-ko mice fed the supplemented diet exhibited equal levels of serum TSH and TT4 compared with the same age of wildtype mice (all $p>0.05$ ). The body weight of $T s h r$-ko mice were significantly lower than wild-type mice $(p<$ 0.05 ), however, the ratio of organ weight to body weight (including the liver, heart and kidney) in $T s h r$-ko mice did not show significant differences compared with wild-type mice (all $p>0.05$ ). To estimate the potential effect of $T s h r$ deletion on liver function, liver histology were observed. The results showed that liver function was normal in $T s h r$-ko mice relative to wildtype mice based on the findings that liver H\&E staining did not show obvious changes (Fig. 1A). Moreover, no
Table 2 General characterization of $T s h r$-ko mice

\begin{tabular}{lcc}
\hline Group & Wild-type & Tshr-knockout \\
\hline BW $(\mathrm{g})$ & $23.77 \pm 1.70$ & $18.76 \pm 1.99^{*}$ \\
Liver weight $(\%$ of BW) & $3.93 \pm 0.50$ & $3.82 \pm 0.23$ \\
Heart weight $(\%$ of BW) & $0.51 \pm 0.08$ & $0.57 \pm 0.08$ \\
Kidney weight $(\%$ of BW) & $1.26 \pm 0.08$ & $1.27 \pm 0.19$ \\
TT4 $(\mu \mathrm{g} / \mathrm{dL}, 6 \mathrm{wk})$ & $5.03 \pm 0.24$ & $4.69 \pm 0.78$ \\
TT4 $(\mu \mathrm{g} / \mathrm{dL}, 8 \mathrm{wk})$ & $3.96 \pm 1.57$ & $4.36 \pm 1.17$ \\
TSH $(\mathrm{pg} / \mathrm{mL})$ & $464.20 \pm 103.37$ & $492.49 \pm 198.04$ \\
\hline
\end{tabular}

Values are given as means \pm SD. The data were analyzed by Student's $t$ test. $\mathrm{n}=6 \sim 8$ animals /group. BW, body weight; TT4, total T4*p $<0.05$

significant differences were observed in plasma insulin (Fig. 1B), glucagon (Fig. 1C) and IGF-1 concentrations (Fig. 1D) between $T s h r$-ko mice and wild-type mice (all $p>0.05$ ), but the level of fasting glucose was reduced to $69 \%$ in $T s h r$-ko mice ( $p<0.01$, Fig. $1 \mathrm{E})$. In line with decreased fasting glucose, $T s h r$-ko mice had increased insulin sensitivity index relative to wild-type mice $(p<0.01$, Fig. 1F).

A
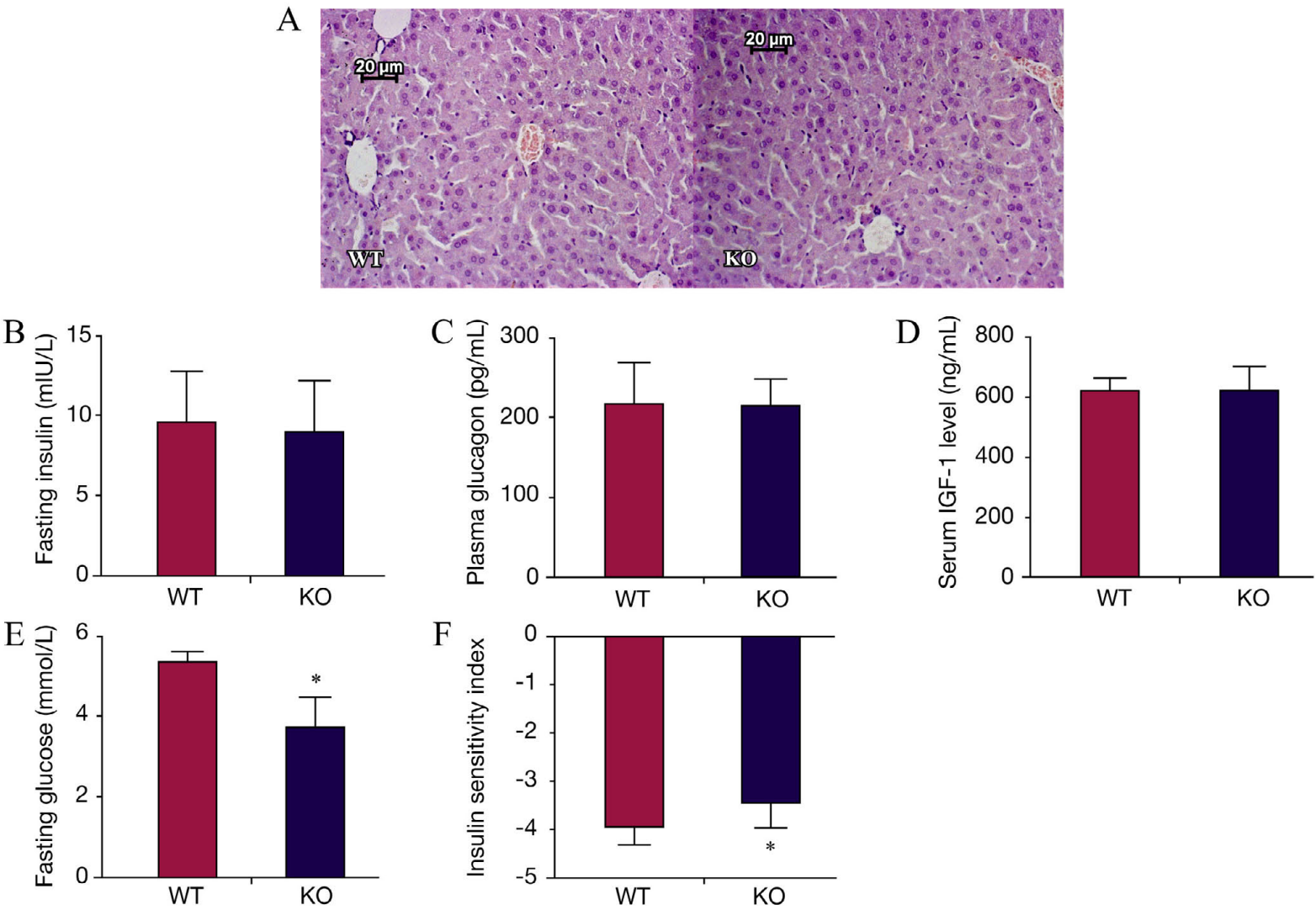

Fig. 1 Histology of liver and glucose metabolic parameters in $T s h r$-ko mice

Wild-type (WT) and Tshr-ko (KO) aged 8-week-old littermate male mice were used. (A) The sections of liver tissues were stained with H\&E, and representative sections are shown. Magnification, $\times 200$. (B, C and D) Fasting plasma insulin, glucagon and IGF-1 concentrations were detected using RIA. (E) Fasting blood glucose was measured with glucometer. (F) Insulin sensitivity was assessed with the insulin sensitivity index. Results are presented as mean \pm SD. $\mathrm{n}=8 \sim 10$ animals/group. ${ }^{*} p<0.01$ 

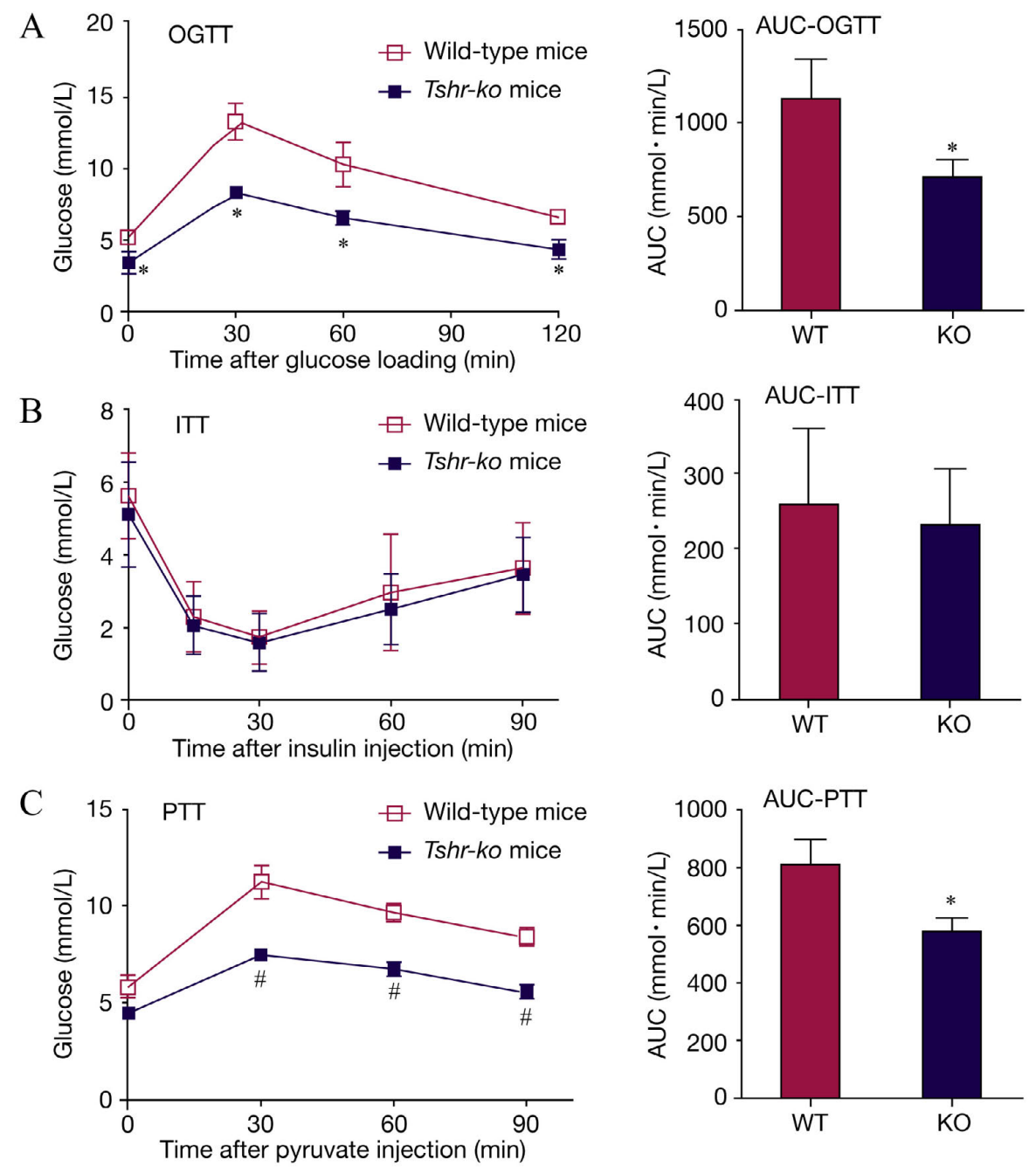

Fig. 2 Tshr deletion improves glucose tolerance and reduces gluconeogenesis

Time curve of glucose levels and area under the curves (AUC) following oral glucose tolerance test (OGTT, A), intraperitoneal insulin tolerance test (ITT, B) and pyruvate tolerance test (PTT, C) in wild-type (WT) and Tshr-ko (KO) mice, respectively. Data are presented as means $\pm \mathrm{SD}, \mathrm{n}=3 \sim 7$ animals/group. ${ }^{*} p<0.05, \# p<0.01$

\section{Tshr deletion improves glucose tolerance and reduces hepatic gluconeogenesis}

To evaluate the effect of $T s h r$ deletion on whole body glucose metabolism, we performed OGTT to observe glucose clearance, ITT to observe insulin-stimulated glucose disposal and PTT to observe gluconeogenesis. In OGTT (Fig. 2A), Tshr-ko mice displayed lower levels of blood glucose throughout the test compared with wild-type mice (all $p<0.05$ ). At $30 \mathrm{~min}$ after glucose loading, the blood glucose level in $T s h r$-ko mice was $62 \%$ that of wild-type mice $(p<0.05)$. In ITT (Fig. 2B), both groups showed the lowest level of blood glucose at $30 \mathrm{~min}$ after the i.p. insulin injection, and the glucose level at $30 \mathrm{~min}$ was reduced to approximately $40 \%$ of glucose at $0 \mathrm{~min}$, which revealed that the amount of insulin injected was appropriate. However, neither time point showed a significant difference in blood glucose throughout the test when compared the two types of mice (all $p>0.05$ ). PTT has been identified as an indicator to reflect changes in hepatic glucose production because pyruvate is a substrate for glucose production and the liver is a major organ for gluconeogenesis [24, 25]. In PTT, both groups achieved peak blood glucose levels at $30 \mathrm{~min}$ after the i.p. pyruvate injection. And $T s h r$-ko mice showed significantly lower glucose levels than wild-type mice at all time points after the i.p. pyruvate injection (all $p<0.01$, Fig. 2 C). The AUC results also supported that $T s h r$ deletion improved pyruvate tol- 


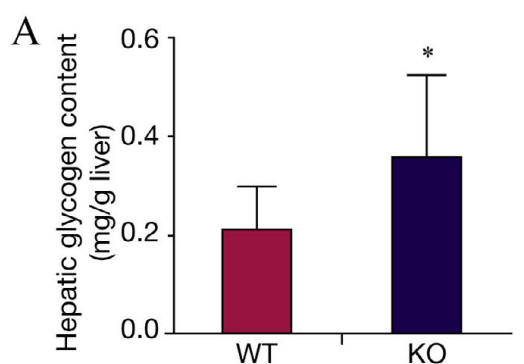

B

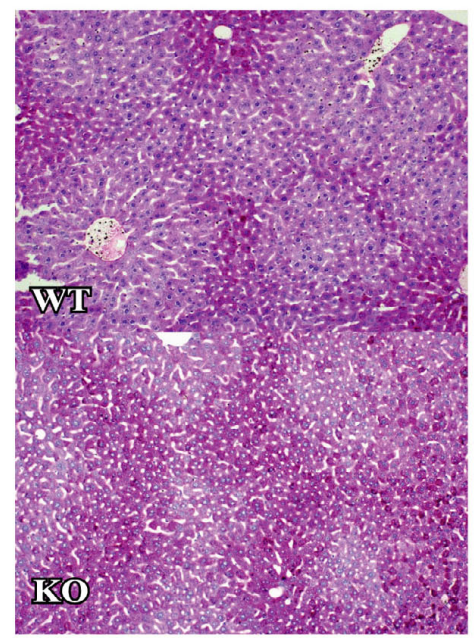

$\mathrm{C}$

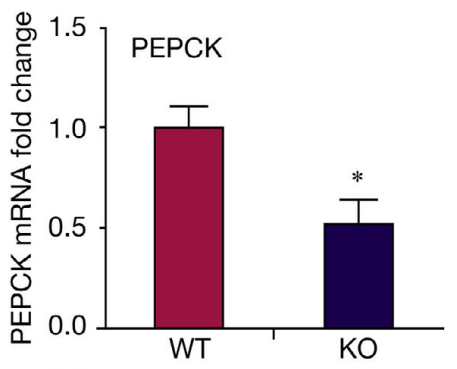

$\mathrm{D}$

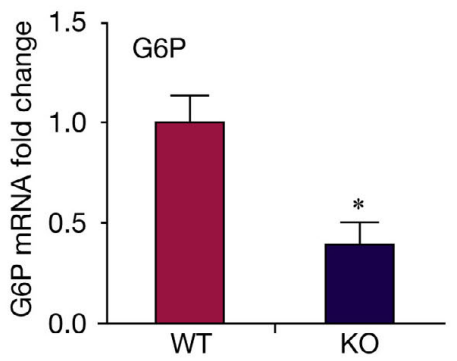

$\mathrm{E}$

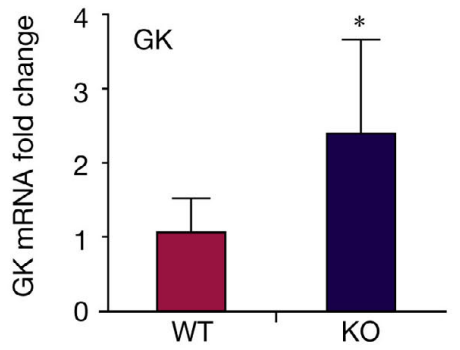

Fig. 3 Down-regulation of fasting-induced gluconeogenesis gene expression in the liver of $T s h r$-ko mice

(A) Liver glycogen content was measured using anthrone reagent. (B) Livers sections from wild-type mice (WT) and Tshr-ko mice (KO) were stained using PAS method. Magnification, $\times 100$. (C, D and E) The expression of hepatic PEPCK, G6P and GK mRNA were determined with quantitative real-time PCR. Results are presented as mean \pm SD. $\mathrm{n}=8 \sim 10$ animals/group. ${ }^{*} p<0.05$

erance, as represented by a significant reduction in the AUC of $T s h r$-ko mice compared with wild-type mice (approximately $25 \%, p<0.05$, Fig. 2 C).

\section{Increased hepatic glycogen contents in Tshr-ko mice}

PAS staining and glycogen assay were performed to evaluate the effect of $T s h r$ deletion on hepatic glycogen storage. The hepatic glycogen assay showed a significant increase (approximately 70\%) in Tshr-ko mice compared with wild-type mice $(p<0.05$, Fig. $3 \mathrm{~A})$. Prunosus particles representing glycogen were observed in the hepatocytic cytoplasm. Consistent with the glycogen content assay result, hepatic glycogen staining also showed a significant augmentation of glycogen storage in $T s h r$-ko mice (Fig. 3B).

\section{Effects of TSH on gluconeogenic gene expression}

To investigate the effect of TSH on the expression of the genes involved in glucose production, the hepatic expression levels of PEPCK and G6P mRNA were ana- lyzed to assess whether TSH influence gluconeogenic gene expression by using real-time PCR. Compared to wild-type mice, the expression levels of PEPCK and G6P mRNA were reduced by $36 \%$ and $47 \%$, respectively, in $T s h r$-ko mice (Fig. 3C \& 3D, all $p<0.05$ ). Moreover, we also determined the abundance of hepatic glucokinase (GK). The results showed that the expression level of GK mRNA was 1.38-fold increase in $T s h r$-ko mice (Fig. 3E, $p<0.05$ ). In HepG2 cells, the abundance of PEPCK and G6P mRNA were significantly increased to $268 \%$ and $159 \%$ after 20 -hours exposure of TSH, respectively, suggesting a direct action of TSH on gluconeogenic gene expression (Fig. 4 , all $p<0.05$ ).

\section{Effects of Tshr deletion on proteins involved in regu- lating hepatic glucose metabolism}

Previous studies indicated that CREB is a downstream molecule of TSH and that it plays a vital role in promoting PEPCK and G6P mRNA expression [13, 
A

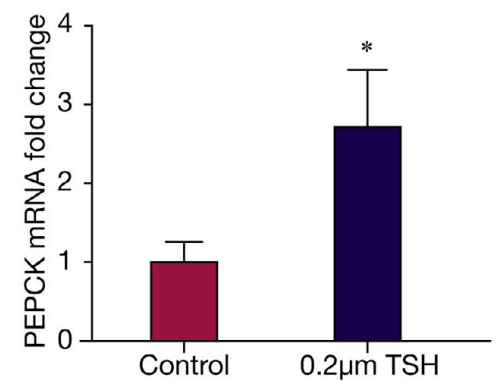

B

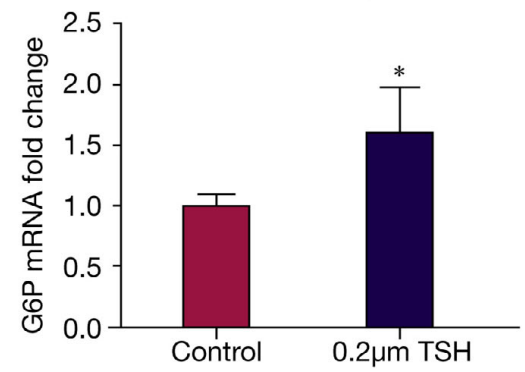

Fig. 4 Up-regulation of PEPCK and G6P mRNA in HepG2 cells by TSH stimulation

(A and B) The effect of TSH $(0.2 \mu \mathrm{m})$ on PEPCK and G6P expression at mRNA levels in HepG2 cells. ${ }^{*} p<0.05$
26]. Therefore, we evaluated the protein expression of p-CREB and CREB in $T s h r$-ko mice. In parallel with the changes in PEPCK and G6P mRNA, the expression of phospho-CREB was diminished in $T s h r$-ko mice ( $p$ $<0.05$ ), while the expression of total CREB was unaltered $(p>0.05)$ (Fig. 5A).

AMPK is a key regulatory protein in glucose signaling transduction. The expression of total AMPK showed no significant difference between $T s h r$-ko mice and wild-type mice (Fig. 5B, $p>0.05$ ). However, the expression level of phospho-AMPK was significantly increased in the liver of $T_{s} h r$-ko mice (Fig. 5B, $p<0.05$ ). Moreover, as one of the downstream target proteins of AMPK, the expression level of phospho-GSK3 $\beta$ was also enhanced in $T s h r$-ko mice (Fig. 5C, $p<0.05$ ).

\section{Discussion}

In the present study, we confirmed the association between TSH and hepatic glucose metabolism for the
A

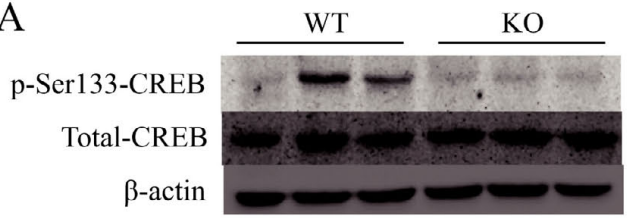

B

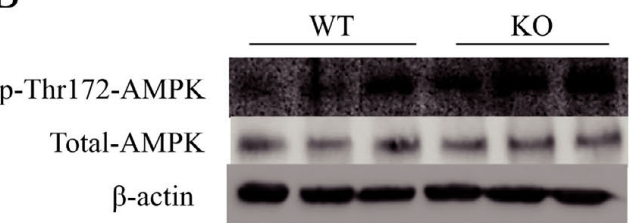

C

p-Ser9-GSK3 $\beta$

Total-GSK3 $\beta$

$\beta$-actin

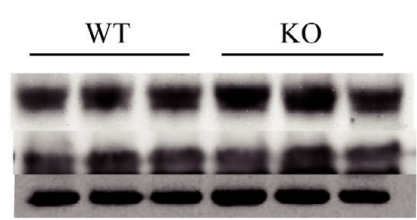

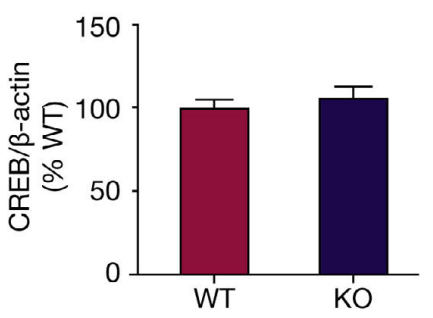
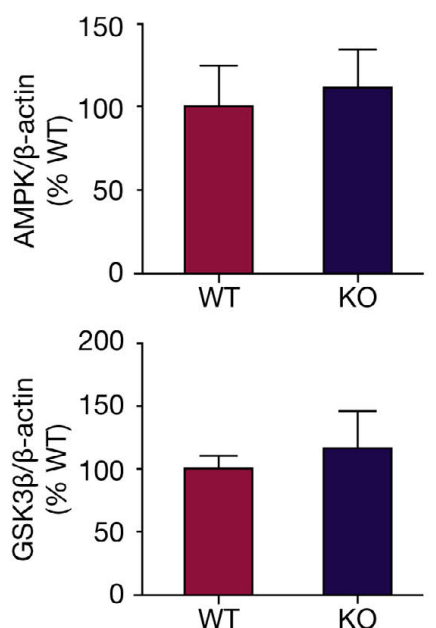
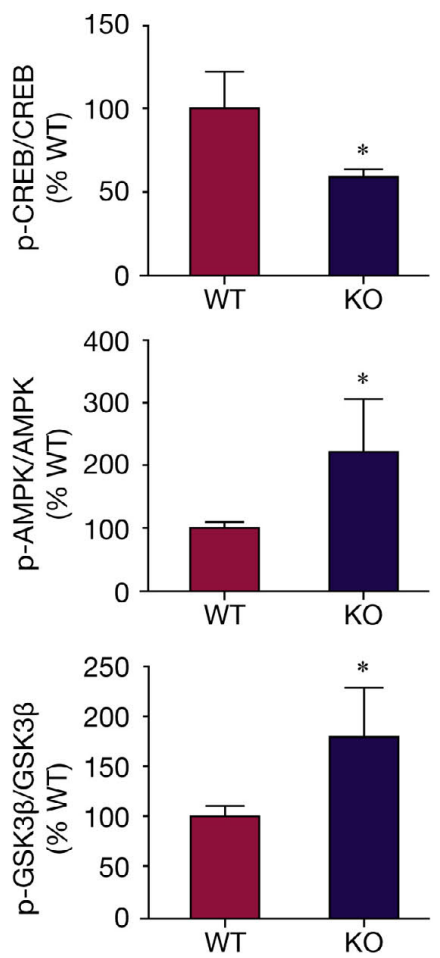

Fig. 5 Effects of Tshr deletion on proteins involved in regulating hepatic glucose metabolism

The total and phosphorylated protein expression of CREB (A), AMPK (B) and GSK3 $\beta$ (C) in the liver of wild-type (WT) and $T s h r$-ko (KO) littermate mice were detected by Western blotting. The relative total protein levels in $T s h r$-ko mice were quantified by densitometry, normalized with $\beta$-actin, and expressed as a value relative to that of wild-type mice. And the relative phosphorylated protein levels in $T s h r$-ko mice were normalized with the corresponding total protein level and expressed as a value relative to that of wild-type mice. Data represent the results of three independent experiments. The data are presented as the mean $\pm \mathrm{SD} . * p<0.05$ 
first time. We found that $T s h r$ deletion in mice elicited fasting hypoglycemia and enhanced insulin sensitivity but did not change plasma insulin levels. The decreased fasting glucose level was due to abnormal hepatic glucose production, including a decrease in hepatic PEPCK and G6P mRNA and an increase in hepatic GK mRNA, and we also found that TSH directly up-regulates the mRNA abundance of gluconeogenic genes in vitro. Moreover, our data indicated that the underlying mechanism of fasting hypoglycemia in $T s h r$-ko mice may be related to the inhibition of hepatic phospho-CREB and the activation of hepatic AMPK.

In the fasting state, glucagon promotes hepatic glucose production and has a hyperglycemic effect via activating enzymes required for gluconeogenesis. Our findings showed a normal level of fasting serum glucagon in $T s h r$-ko mice, making it unlikely that the decreased fasting glucose was due to altered glucagon level. And we also eliminate the effect of IGF-1 which could reduce blood glucose level based on the finding that there was no significant difference of IGF-1 level between 8-week-old $T s h r$-ko mice and wild-type mice. However, Xing et al. [27] has reported lower IGF-1 concentrations in their $T s h r$-ko mice. The difference maybe due to the different age of mice measured the serum IGF-1 level. Xing measured the level of serum IGF-1 when the mice were 21 days old. However, we measured at the age of 8 weeks. Our data implied that Tshr deletion did not affect serum IGF-1 level at the age of 8 weeks. Moreover, we eliminated the negative effects of liver injury on hepatic gluconeogenesis because of the generally normal liver histology in $T s h r$-ko mice compared with wild-type mice.

Hepatic glucose output is vital for maintaining fasting glucose homeostasis. In the fasted state, liver produces glucose by either gluconeogenesis or glycogenolysis to provide energy for maintaining normal functions. PEPCK and G6P are rate-limiting enzymes that control hepatic gluconeogenesis [28], and G6P also regulates the last step of glycogenolysis. The transcription of PEPCK and G6P mRNA determines the rate of hepatic gluconeogenesis [29]. In $T s h r$-ko mice, the decreased gene expression of liver PEPCK and G6P mRNA indicated a reduced capacity of hepatic gluconeogenesis and glycogenolysis. Consistent with these results, a lower AUC of the pyruvate tolerance test and increased storage of liver glycogen were observed in Tshr-ko mice. To estimate the effect of TSH on gluconeogenesis in vitro, the expression of PEPCK and G6P
mRNA in HepG2 cells were determined after stimulation by TSH. The results confirmed a direct action of TSH on gluconeogenic genes in HepG2 cells. Hepatic gluconeogenic gene expression is mediated by multiple transcriptional factors, such as CREB, which has binding sites in the promoter of PEPCK and G6P genes [26]. In our study, we observed Tshr deletion leading to the down-regulation of phospho-CREB expression, but the expression of total CREB was unchanged, suggesting that $T s h r$ deletion may impair hepatic glucose production partly by inhibiting CREB phosphorylation.

Recently, several studies reported that long-term AMPK activation could directly repress the gene expression of PEPCK and G6P and inhibit hepatic glucose output [30]. And TSH has been reported to inhibit the phosphorylation of AMPK in the thyroid gland [17]. To determine whether Tshr deletion influenced AMPK activity in the liver, we measured the protein expression of hepatic phospho-AMPK. We found that phospho-AMPK expression was significantly increased in $T s h r$-ko mice. As AMPK downstream target protein, in parallel with phospho-AMPK, the expression of phospho-GSK3 $\beta$ was also enhanced in $T s h r$-ko mice. Increased GSK $3 \beta$ phosphorylation plays a critical role in AMPK-induced suppression of hepatic gluconeogenesis [16]. Moreover, GSK3 $\beta$ is also an upstream inhibitor of glycogen synthesis. As the first enzyme in glycogen synthesis, GK was markedly increased determined by the level of GK mRNA in the liver of Tshr-ko mice. These results indicated that the increased glycogen storage in the liver of $T s h r$-ko mice could be explained not only by reduced glycogenolysis, but also by enhanced glycogen synthesis. Taken together, the changes of gluconeogenesis, glycogenolysis, and glycogen synthesis in the liver of $T s h r$-ko mice were partly due to the increase of AMPK activation. Furthermore, it was well known that activation of AMPK could enhance insulin sensitivity. Hepatic AMPK activation explains the improvement in insulin sensitivity observed in $T s h r$-ko mice. However, we did not observe obvious glucose changes between the two groups in the insulin tolerance test. One possible explanation was that the insulin tolerance test is a crude technique to estimate insulin-stimulated glucose disposal because rapid declines in glucose usually lead to changes in many hormones regulating glucose metabolism during the insulin tolerance test [31]. As a result, the glucose levels of the insulin tolerance test may be due to a complex interaction of hormones, not only the action of insulin. In our 
study, a more accurate technique, such as a hyperinsulinemic-euglycemic clamp, is needed to quantitatively assess insulin sensitivity. This was a limitation of our study. However, a previous report has shown that ISI is significantly correlated with the glucose turnover rate measured by a hyperinsulinemic-euglycemic clamp ( $\mathrm{r}$ $>0.7, p=0.0001$ ) [19], which suggests that ISI can be a simple method for estimating insulin sensitivity to some extent.

Thyroxine (T4) stimulates hepatic gluconeogenesis, and even a small change in T4 levels can affect glucose metabolism [32]. In our study, we fed $T s h r$-ko mice supplemented diet to eliminate the effect of T4 on hepatic gluconeogenesis. We found that both 6 -weekold and 8-week-old Tshr-ko mice have equivalent serum TT4 levels with that of wild-type mice at the same age (Table 2), indicating that the supplement diet was appropriate for the $T s h r$-ko mice at the age of 6-8 weeks. Although there was a slightly higher level of serum TT4 in 8-week-old Tshr-ko mice, the differences in serum TT4 and TSH levels were not statistically significant between the two types of mice. In addition, a higher serum TT4 level in Tshr-ko mice was hypothesized to elevate fasting glucose, which was in contrast to the fasting hypoglycemia observed in Tshr-ko mice. Taken together, these data indicated that we could ignore the extra effect of T4 on hepatic gluconeogenesis when comparing $T s h r$-ko mice with wild- type mice.

Our study confirmed a novel role for TSH in hepatic glucose metabolism. The inhibition of hepatic PEPCK and G6P and enhanced expression of GK contributed to the development of fasting hypoglycemia in $T s h r$-ko mice. And TSH had direct impact on gluconeogenesis in vitro. Tshr deletion affected the expression of proteins involved in regulating hepatic glucose production. Further studies are needed to elucidate how TSH affects AMPK activation and whether other insulin target organs are involved in reducing fasting glucose. Our study provides possible pathological implications for the pathogenesis of abnormal glucose metabolism in $\mathrm{SCH}$ patients.

\section{Acknowledgements}

This work was supported by grants from the National Basic Research Program (2012CB524900), the National Natural Science Foundation (30971409, 81230018, 81170794, 81270869 and 81270970), the Natural Science Foundation (2012GSF11824), Provincial research fund for excellent young and middle-aged scientists of Shandong Province (BS2011SW034), Provincial Natural Science Fund (ZR2009CM101), an international cooperation grant (2011) of Shandong Province and the Jinan self-renovation plan for colleges, universities and scientific research institutes (2012) of China.

\section{References}

1. Turhan S, Sezer S, Erden G, Guctekin A, Ucar F, et al. (2008) Plasma homocysteine concentrations and serum lipid profile as atherosclerotic risk factors in subclinical hypothyroidism. Ann Saudi Med 28: 96-101.

2. Asvold BO, Bjoro T, Platou C, Vatten LJ (2012) Thyroid function and the risk of coronary heart disease: 12-year follow-up of the HUNT Study in Norway. Clin Endocrinol (Oxf) 77: 911-917.

3. Dessein PH, Joffe BI, Stanwix AE (2004) Subclinical hypothyroidism is associated with insulin resistance in rheumatoid arthritis. Thyroid 14: 443-446.

4. Al Sayed A, Al Ali N, Bo Abbas Y, Alfadhli E (2006) Subclinical hypothyroidism is associated with early insulin resistance in Kuwaiti women. Endocr J 53: 653657.

5. Maratou E, Hadjidakis DJ, Kollias A, Tsegka K, Peppa M, et al. (2009) Studies of insulin resistance in patients with clinical and subclinical hypothyroidism. Eur $J$ Endocrinol 160: 785-790.

6. Tuzcu A, Bahceci M, Gokalp D, Tuzun Y, Gunes K
(2005) Subclinical hypothyroidism may be associated with elevated high-sensitive c-reactive protein (low grade inflammation) and fasting hyperinsulinemia. Endocr J 52: 89-94.

7. Amati F, Dube JJ, Stefanovic-Racic M, Toledo FG, Goodpaster BH (2009) Improvements in insulin sensitivity are blunted by subclinical hypothyroidism. Med Sci Sports Exerc 41: 265-269.

8. Velija-Asimi Z, Karamehic J (2007) The effects of treatment of subclinical hypothyroidism on metabolic control and hyperinsulinemia. Med Arh 61: 20-21.

9. Kowalska I, Borawski J, Nikolajuk A, Budlewski T, Otziomek E, et al. (2011) Insulin sensitivity, plasma adiponectin and sICAM-1 concentrations in patients with subclinical hypothyroidism: response to levothyroxine therapy. Endocrine 40: 95-101.

10. Peeters RP, van der Deure WM, van den Beld AW, van Toor H, Lamberts SW, et al. (2007) The Asp727Glu polymorphism in the TSH receptor is associated with insulin resistance in healthy elderly men. Clin Endocrinol 66: 
808-815.

11. Davies T, Marians R, Latif R (2002) The TSH receptor reveals itself. J Clin Invest 110: 161-164.

12. Zhang W, Tian LM, Han Y, Ma HY, Wang LC, et al. (2009) Presence of thyrotropin receptor in hepatocytes: not a case of illegitimate transcription. J Cell Mol Med 13: 4636-4642.

13. Tian L, Song Y, Xing M, Zhang W, Ning G, et al. (2010) A novel role for thyroid-stimulating hormone: up-regulation of hepatic 3-hydroxy-3-methyl-glutaryl-coenzyme A reductase expression through the cyclic adenosine monophosphate/protein kinase A/cyclic adenosine monophosphate-responsive element binding protein pathway. Hepatology 52: 1401-1409.

14. Staehr P, Hother-Nielsen O, Beck-Nielsen H (2004) The role of the liver in type 2 diabetes. Rev Endocr Metab Disord 5: 105-110.

15. Viollet B, Lantier L, Devin-Leclerc J, Hebrard S, Amouyal C, et al. (2009) Targeting the AMPK pathway for the treatment of Type 2 diabetes. Front Biosci 14: 3380-3400.

16. Horike N, Sakoda H, Kushiyama A, Ono H, Fujishiro $\mathrm{M}$, et al. (2008) AMP-activated protein kinase activation increases phosphorylation of glycogen synthase kinase 3 beta and thereby reduces cAMP-responsive element transcriptional activity and phosphoenolpyruvate carboxykinase $\mathrm{C}$ gene expression in the liver. $\mathrm{J}$ Biol Chem 283: 33902-33910.

17. Andrade BM, Araujo RL, Perry RL, Souza EC, Cazarin $\mathrm{JM}$, et al. (2011) A novel role for AMP-kinase in the regulation of the $\mathrm{Na}+/ \mathrm{I}-$-symporter and iodide uptake in the rat thyroid gland. Am J Physiol Cell Physiol 300: C1291-1297.

18. Marians RC, Ng L, Blair HC, Unger P, Graves PN, et al. (2002) Defining thyrotropin-dependent and -independent steps of thyroid hormone synthesis by using thyrotropin receptor-null mice. Proc Natl Acad Sci U S A 99: 15776-15781.

19. Li GW, Pan XR (1993) A new insulin-sensitivity index for the population-based study. Zhonghua Nei Ke Za Zhi 32: 656-660 (In Chinese).

20. Taniguchi CM, Kondo T, Sajan M, Luo J, Bronson R, et al. (2006) Divergent regulation of hepatic glucose and lipid metabolism by phosphoinositide 3-kinase via Akt and PKClambda/zeta. Cell Metab 3:343-353.

21. Miyake K, Ogawa W, Matsumoto M, Nakamura T, Sakaue H, et al. (2002) Hyperinsulinemia, glucose intolerance, and dyslipidemia induced by acute inhibition of phosphoinositide 3-kinase signaling in the liver. J Clin Invest 110: 1483-1491.

22. Livak KJ, Schmittgen TD (2001) Analysis of relative gene expression data using real-time quantitative PCR and the 2(-Delta Delta C(T)) Method. Methods 25: 402408.

23. Seifter S, Dayton S, et al. (1950) The estimation of glycogen with the anthrone reagent. Arch Biochem 25:191200.

24. Rodgers JT, Puigserver P (2007) Fasting-dependent glucose and lipid metabolic response through hepatic sirtuin 1. Proc Natl Acad Sci US A 104: 12861-12866.

25. Lin J, Wu PH, Tarr PT, Lindenberg KS, St-Pierre J, et al. (2004) Defects in adaptive energy metabolism with CNS-linked hyperactivity in PGC-1alpha null mice. Cell 119: 121-135.

26. Jitrapakdee S (2012) Transcription factors and coactivators controlling nutrient and hormonal regulation of hepatic gluconeogenesis. Int $J$ Biochem Cell Biol 44: $33-45$.

27. Xing $\mathrm{W}$, Govoni KE, Donahue LR, Kesavan C, Wergedal J, et al. (2012) Genetic evidence that thyroid hormone is indispensable for prepubertal insulinlike growth factor-I expression and bone acquisition in mice. J Bone Miner Res 27: 1067-1079.

28. Pilkis SJ, Granner DK (1992) Molecular physiology of the regulation of hepatic gluconeogenesis and glycolysis. Annu Rev Physiol 54: 885-909.

29. Goto M, Yoshioka T, Battelino T, Ravindranath T, Zeller WP (2001) TNFalpha decreases gluconeogenesis in hepatocytes isolated from 10-day-old rats. Pediatr Res 49: 552-557.

30. Viollet B, Foretz M, Guigas B, Horman S, Dentin R, et al. (2006) Activation of AMP-activated protein kinase in the liver: a new strategy for the management of metabolic hepatic disorders. J Physiol 574: 41-53.

31. Brehm A, Thomaseth K, Bernroider E, Nowotny P, Waldhausl W, et al. (2006) The role of endocrine counterregulation for estimating insulin sensitivity from intravenous glucose tolerance tests. J Clin Endocrinol Metab 91: 2272-2278.

32. Comte B, Vidal H, Laville M, Riou JP (1990) Influence of thyroid hormones on gluconeogenesis from glycerol in rat hepatocytes: a dose-response study. Metabolism 39: 259-263. 